\title{
Orthostatic hypotension: does the heart rate matter? And other updates on recent autonomic research
}

\author{
Mitchell G. Miglis ${ }^{1} \cdot$ Srikanth Muppidi ${ }^{1,2}$
}

Received: 8 May 2018 / Accepted: 10 May 2018 / Published online: 19 May 2018

(c) Springer-Verlag GmbH Germany, part of Springer Nature 2018

Keywords Neurogenic orthostatic hypotension · Familial dysautonomia · Antisense oligonucleotide therapy

\section{Orthostatic hypotension: does the heart rate matter?}

Orthostatic hypotension $(\mathrm{OH})$ can be due to neurogenic and non-neurogenic causes. Non-neurogenic causes include low plasma volume and severe physical deconditioning, and are usually associated with compensatory tachycardia, in contrast to neurogenic orthostatic hypotension $(\mathrm{nOH})$ in which there is a minimal change in heart rate (HR) despite significant hypotension. $\mathrm{OH}$ is currently defined as a sustained drop of at least $20 \mathrm{mmHg}$ in systolic or $10 \mathrm{mmHg}$ in diastolic blood pressure (BP). The current consensus statement for the definition of $\mathrm{OH}$ [1] suggests an increase in $\mathrm{HR}$ of $<15$ beats per minute (bpm) in spite of hypotension as a marker of nOH. However, this statement was based on the opinion of autonomic experts and not on scientific evidence.

To further address the definition of $\mathrm{OH}$, the US Autonomic Disorders Consortium recently published their analysis of orthostatic HR changes in a cohort of patients with $\mathrm{nOH}$ due to neurodegenerative synucleinopathies, including multiple system atrophy (MSA), Parkinson disease (PD), dementia with Lewy bodies (DLB), and pure autonomic failure (PAF) [2], enrolled in the Natural History Study of the Synucleinopathies. All patients underwent standardized clinical evaluation and autonomic testing, including the tilt-table test, Valsalva maneuver, and measures of HR

This comment refers to three articles available at: https:// doi.org/10.1002/ana.25170; https://doi.org/10.1016/j.sleep .2017.12.013; https://doi.org/10.1007/s00109-006-0137-2.

Srikanth Muppidi

muppidis@stanford.edu

Stanford Medical Center, Palo Alto, CA, USA

2 Stanford Neurosciences Health Center, 213 Quarry Road, 2nd Floor, Palo Alto, CA 94304, USA variability with paced breathing. The authors identified 402 patients with $\mathrm{OH}$. Of these, 378 patients had $\mathrm{nOH}$ and were diagnosed with one of the synucleionopathies. The remaining 24 had preserved sympathetic function and were diagnosed with non-neurogenic $\mathrm{OH}$. Patients in the $\mathrm{nOH}$ group were then further subdivided into those with central autonomic failure ( $n=96$; diagnosed with MSA) and peripheral autonomic failure $(n=128$; including those diagnosed with DLB, PD, and PAF without signs of central nervous system [CNS] involvement, such as anosmia or REM behavior disorder) and prodromal disease ( $n=154$; PAF with signs of CNS involvement).

Patients with $\mathrm{nOH}$ had a significantly greater fall in systolic BP (SBP; $-44 \pm 25$ vs. $-21 \pm 14 \mathrm{mmHg}$ [mean \pm standard deviation]; $p<0.0001)$ at $3 \mathrm{~min}$ of head-up tilt testing. As expected, the HR increase was much lower in the $\mathrm{nOH}$ group than in the non-neurogenic $\mathrm{OH}$ group $(8 \pm 8$ vs. $25 \pm 11 \mathrm{bpm} ; p<0.0001]$. Since there was a significant overlap in HR and BP changes in the neurogenic and nonneurogenic groups, the researchers also calculated the ratio of HR change over SBP change (i.e., $\Delta \mathrm{HR} / \Delta \mathrm{SBP}$ ratio). A $\Delta \mathrm{HR} / \Delta \mathrm{SBP}$ ratio cut-off of $0.492 \mathrm{bpm} / \mathrm{mmHg}$ had excellent sensitivity (91.3\%) and specificity $(88.4 \%)$ to distinguish between patients with neurogenic and non-neurogenic $\mathrm{OH}$.

In addition, patients with central autonomic failure only (MSA) had a greater increase in HR than did patients with peripheral autonomic failure. However, due to overlapping results, the change in HR could not distinguish between central and peripheral $\mathrm{nOH}$. More importantly, the $\Delta \mathrm{HR} /$ $\Delta$ SBP ratio was not different in patients with central versus peripheral $\mathrm{nOH}$.

This study has a few limitations, including the fact that all of the BP and HR data were obtained from tilt-table testing. Since there are likely some differences between active standing (commonly used in clinics for evaluating patients with $\mathrm{OH}$ ) and passive standing (tilt-table testing), the $\Delta \mathrm{HR} /$ 
$\Delta$ SBP ratio might be different with active standing. In addition, autonomic centers also perform Valsalva testing with tilt-table studies, and various sympathetic measures during Valsalva testing are likely reliable markers of sympathetic failure [3]. If these findings can be replicated in bedside active stand testing, it will clearly help clinicians evaluate the patient for $\mathrm{nOH}$ during routine clinic visits and perhaps can become the standard of care for patients susceptible to orthostatic syndromes.

\section{Sleep disordered breathing in familial dysautonomia: a risk factor of central importance}

Familial dysautonomia (FD; also known as Riley-Day syndrome and hereditary sensory and autonomic neuropathy [HSAN] type III), is a rare autosomal recessive sensory and autonomic neuropathy that affects mostly children of Jewish Ashkenazi descent. FD is caused by a founder mutation in the IkB kinase-associated protein (IKBKAP) gene, which leads to incomplete development of sensory afferent neurons throughout the somatic and autonomic nervous system. As a result, FD patients have impaired pain and temperature sensation, baroreflex failure with BP and HR instability, and sleep-disordered breathing, among other comorbid conditions. Sudden unexpected death can occur in sleep, and the presence of untreated sleep-disordered breathing (SDB) has been shown to increase this risk [4]. For these reasons, it is thought that the de-afferentation of autonomic nerves might play a role in the sleep-related respiratory instability and mortality risk in patients with FD.

In their manuscript entitled "Prevalence and characteristics of SDB in familial dysautonomia," published in the May 2018 edition of Sleep Medicine, Singh and colleagues evaluated the type and severity of SDB in a group of 75 carefully phenotyped patients with FD [5]. The authors enrolled a consecutive series of genetically-confirmed patients homozygous for the IKBKAP founder mutation, regardless of sleep-related symptoms. All patients underwent an in-lab polysomnogram to evaluate for the presence of obstructive sleep apnea (OSA) and central sleep apnea (CSA). Apneas and hypopneas were manually scored and classified into mild, moderate, and severe based on the apnea-hypopnea index (AHI). Hypopneas were scored based on a desaturation of $4 \%$ for adults and 3\% for children, and average and peak $\mathrm{EtCO}_{2}$ (partial pressure or maximal concentration of carbon dioxide $\left[\mathrm{CO}_{2}\right]$ at the end of an exhaled breath) values were reported. In children (age $<18$ years), an AHI of $1-5$ was classified as mild, $6-10$ as moderate, and $>10$ as severe. In adults (age $>18$ years), an AHI of $<5$ was classified as normal, 5-14 as mild, $15-30$ as moderate, and $>30$ as severe.
The authors found that $85 \%$ of adults and $91 \%$ children in their cohort met criteria for SDB, with the majority of patients falling into the mild category. OSA occurred in $47 \%$ of patients ( $45 \%$ of children and $50 \%$ of adults), and CSA occurred in $57 \%$ of patients ( $62 \%$ of children and $45 \%$ of adults). Of the 35 patients with OSA, $60 \%$ had mild, $26 \%$ had moderate, and $14 \%$ had severe OSA. Of the 43 patients with central apneas, $70 \%$ had mild, $12 \%$ had moderate, and $19 \%$ had severe central apneas. All central apneas occurred in all stages of sleep, independent of any fragmented sleep or arousals.

Hypoventilation, defined as a maximum $\mathrm{EtCO}_{2}$ level of $>50 \mathrm{mmHg}$ in children and $>55 \mathrm{mmHg}$ in adults, occurred in $62 \%$ of children and in $60 \%$ of adults, and $46 \%$ of all patients experienced hypoventilation with no accompanying sleep apnea. OSA was more frequent in adult patients, whereas CSA was more frequent in children.

These results indicate that the mechanisms driving CSA tend to have less influence as the patients aged, suggesting that the developing brain is more prone to CSA, especially in those with FD. The limitations of this study include the lack of a control group, the fact that the authors did not control for body mass index or the prevalence of comorbid cardiopulmonary diagnoses (e.g., laryngo-tracheobronchomalacia, congenital heart defects), and the lack of a prospective follow-up to determine the relevance of the authors' findings. These limitations, coupled with lack of correlation with symptoms, make the clinical significance of these results somewhat difficult to interpret. Most patients' SDB was mild, and thus without longitudinal follow-up data, it is hard to draw conclusive associations with morbidity and mortality risk.

Nonetheless, this study includes the largest cohort of patients with FD and polysomnography data to date. The authors demonstrated that a majority of the adult and pediatric patients with FD enrolled in their study suffer from some degree of SDB, with CSA more prevalent in younger patients and OSA more prevalent in older patients. Therefore, any polysomnogram in a patient with FD should include $\mathrm{CO}_{2}$ monitoring. We look forward to the authors' longitudinal outcome data in this very unique population.

\section{Antisense oligonucleotide therapy for familial dysautonomia}

Antisense oligonucleotide (ASO) therapy is currently in development for many neuromuscular diseases [6]. Two ASOs were recently approved by the US Food and Drug Administration (FDA) for the treatment of neuromuscular diseases: eteplirsen for Duchenne muscular dystrophy and nusinersen for spinal muscular atrophy [7]. ASOs are essentially synthetic, short, single-stranded oligodeoxynucleotides 
that can alter RNA and reduce, restore, or modify protein expression through several distinct mechanisms. This therapeutic strategy is ideal in single-gene syndromes. FD is one such disease, caused by a point mutation of intron 20 in the $I K B K A P$ gene. This mutation leads to the skipping of exon 20 , resulting in decreased levels of IKAP protein. The IKAP protein plays a key role in the development and survival of peripheral neurons.

Previous researchers have attempted to increase the IKAP protein levels with various therapies, including treatment with the plant cytokinin kinetin, which alters splicing of $I K B K A P$ and increases inclusion of exon 20, thereby increasing the total amount of IKAP protein [8]. Kinetin is currently undergoing a phase II clinical trial in patients with FD.

ASO therapy is another potential strategy to increase the IKAP protein. Sinha and colleagues set out to study whether ASOs can potentially increase IKAP levels in the fibroblasts of FD patients and in a transgenic FD mouse model. After identifying an appropriate ASO, they observed that this ASO significantly increased IKAP protein levels in fibroblasts derived from a FD patient 3 days after treatment. They then treated transgenic mice models carrying the human FD IKBKAP gene mutation; however this particular mice model does not exhibit any of the FD clinical phenotypes as they also have the wild-type mouse IKBKAP gene. ASO was injected into the ventricular space and subcutaneously. This resulted in a local increase in IKBKAP mRNA levels and an increase in IKAP protein levels. This is very preliminary work, and we still need proof that this ASO-based treatment actually improves the FD phenotype in mouse models. However, there are reasons to be optimistic as current FDAapproved ASO treatments of other neuromuscular diseases followed similar steps only few years ago. We are hopeful that future studies will expand upon this technology and bring us that much closer to a cure for disabling genetic autonomic disorders.
Funding support None

\section{Compliance with ethical standards}

Conflict of interests The authors declare that they have no conflict of interests.

\section{References}

1. Freeman R, Wieling W, Axelrod FB et al (2011) Consensus statement on the definition of orthostatic hypotension, neurally mediated syncope and the postural tachycardia syndrome. Clin Auton Res 21:69-72. https://doi.org/10.1007/s10286-011-0119-5

2. Norcliffe-Kaufmann L, Kaufmann H, Palma JA et al (2018) Orthostatic heart rate changes in patients with autonomic failure caused by neurodegenerative synucleinopathies. Ann Neurol 83:522-531. https://doi.org/10.1002/ana.25170

3. Novak P (2011) Assessment of sympathetic index from the Valsalva maneuver. Neurology 76:2010-2016. https://doi.org/10.1212/ WNL.0b013e31821e5563

4. Palma JA, Norcliffe-Kaufmann L, Perez MA et al (2017) Sudden unexpected death during sleep in familial dysautonomia: a casecontrol study. Sleep. https://doi.org/10.1093/sleep/zsx083

5. Singh K, Palma JA, Kaufmann H et al (2018) Prevalence and characteristics of sleep-disordered breathing in familial dysautonomia. Sleep Med 45:33-38. https://doi.org/10.1016/J.SLEEP .2017.12.013

6. Goyal N, Narayanaswami P (2018) Making sense of antisense oligonucleotides: a narrative review. Muscle Nerve 57:356-370. https://doi.org/10.1002/mus.26001

7. Rinaldi C, Wood MJA (2018) Antisense oligonucleotides: the next frontier for treatment of neurological disorders. Nat Rev Neurol 14:9-21. https://doi.org/10.1038/nrneurol.2017.148

8. Hims MM, Ibrahim EC, Leyne M et al (2007) Therapeutic potential and mechanism of kinetin as a treatment for the human splicing disease familial dysautonomia. J Mol Med 85:149-161. https ://doi.org/10.1007/s00109-006-0137-2 